\title{
Preparation and characterization of silver nanoparticles on silk fibroin/ carboxymethy lchitosan composite sponge as anti-bacterial wound dressing
}

\author{
Zejun Pei ${ }^{\mathrm{a}}$, Qing Sun ${ }^{\mathrm{a}}$, Xin Sun ${ }^{\mathrm{a}}$, Yaping Wang ${ }^{\mathrm{a}}$ and Peng Zhao ${ }^{\mathrm{b}, *}$ \\ ${ }^{a}$ Department of Pharmacy, Wuxi No. 2 People's Hospital, Wuxi 214002, P.R. China \\ ${ }^{b}$ Department of Central Laboratory, Wuxi Third People's Hospital, Wuxi 214122, P.R. China
}

\begin{abstract}
The infection in burn wounds covered by biologic dressings leads to wound deepening and chronic wounds. The introduction of silver nanoparticles (AgNPs) into biologic dressings is a beneficial method to prevent wound infection and simultaneously promote wound healing. In this study, an AgNP-loaded silk fibroin (SF)/carboxymethylchitosan (CMC) composite sponge was fabricated. AgNPs with a mean diameter of $4.9 \mathrm{~nm}$ was synthesized in SF solution in situ. While CMC was incorporated and chemically crosslinked, SF was insolubilized by ethanol annealing. SEM imaging determined that the AgNP-loaded SF/CMC sponge was more porous than the pure SF sponge. Anti-bacterial results, measured by disk-diffusion and bacterial suspension assay, showed that the AgNP-loaded SF/CMC sponge demonstrated effective anti-bacterial activity against $S$. aureus and $P$. aeruginosa, and that its anti- $P$. aeruginosa activity was higher than that of AQUACEL $\AA$ Ag. The introduction of CMC improved the water absorption capacity, retention ability, and water vapor transmission rate of the sponge, which are all important properties of wound dressings.
\end{abstract}

Keywords: Silver nanoparticles, silk fibroin, carboxymethylchitosan, wound infection, wound healing

\section{Introduction}

Biologic dressings are used to create a moist environment for burn wounds and to promote wound repair and regeneration [1]. However, such an environment is also an ideal place for the colonization of pathological bacteria, such as Staphylococcus aureus (S. aureus) and Pseudomonas aeruginosa (P. aeruginosa). Therefore, it is beneficial to develop a novel biologic dressing to promote both wound healing and anti-infection.

Antibiotics or antiseptics have long been encapsulated into and delivered by biologic dressings to prevent wound infection. Functioning as temporary epidermal substitutes, these dressings act as delivery systems for sustained drug release $[2,3]$. However, due to the growing number of antibioticresistant microorganisms, it is contentious to apply topical antibiotics to wound surfaces [4]. As an

\footnotetext{
*Address for correspondence: Peng Zhao, Department of Central Laboratory, Wuxi Third People's Hospital, North Xingyuan Road 585, Wuxi, P.R. China. Tel.: +86 510 81195776; Fax: +86 510 82606599; E-mail: zhaop60@126.com.
} 
anti-bacterial substance, silver has a low probability of resistance and demonstrates broad-spectrum anti-microbial activity [5]. Commercial biologic dressings containing silver ions are widely available, such as AQUACEL ${ }^{\circledR} \mathrm{Ag}$ [6]. Ag nanoparticles (AgNPs) have been encapsulated into various polymeric matrices $[7,8]$. It has been suggested that AgNPs are more effective than other forms of silver in bacterial suppression [9]. Thus, incorporating AgNPs into biologic dressings is a promising strategy in infection prevention and the simultaneous improvement of wound healing.

This study sought to develop a hybrid silk fibroin/carboxymethylchitosan sponge loaded with AgNPs, which is able to prevent wound infection and simultaneously promote wound healing. AgNPs were synthesized in situ in regenerated silk fibroin solution, and then supplemented with carboxymethylchitosan. The AgNP-loaded sponge was prepared by lyophilizing, physical crosslinking of silk fibroin, and chemical crosslinking of carboxymethylchitosan. The anti-bacterial activity of the sponge was measured by disk diffusion and a bacterial suspension assay. Derived from Bombyx mori, silk fibroin demonstrates excellent biocompatibility and robust mechanical strength, but its application as a wound dressing is limited due to its high degree of crystallinity. In this study, in order to improve the internal structure of the silk fibroin-based sponge, carboxymethylchitosan was introduced to decrease the degree of crystallinity of silk fibroin. Therefore, it was hypothesized that the loading of AgNPs could result in a silk fibroin/carboxymethylchitosan hybrid sponge, with sustained antibacterial potential and improved physichemical properties, which is promising as an excellent antibacterial wound dressing.

\section{Materials and methods}

\subsection{Preparation of silk fibroin (SF) solution}

Impurity-removed Bombyx mori silk cocoons were boiled and degummed in $0.02 \mathrm{M} \mathrm{Na}_{2} \mathrm{CO}_{3}$ solution at $100^{\circ} \mathrm{C}$ for 45 minutes, washed five times with distilled water, and then dried overnight at $50^{\circ} \mathrm{C}$ to obtain SF. The resulting SF was dissolved in a ternary solvent, $\mathrm{CaCl}_{2} / \mathrm{CH}_{3} \mathrm{CH}_{2} \mathrm{OH} / \mathrm{H}_{2} \mathrm{O}(1: 2: 8$ molar ratio) at $70^{\circ} \mathrm{C}$ for five hours. The SF solution was then dialyzed against distilled water for three days. The fibroin concentration was determined by analytical balance after undergoing water evaporation overnight.

\subsection{Preparation and characterization of AgNPs in SF solution}

Fresh $\mathrm{AgNO}_{3}$ solution $(0.05 \mathrm{wt} \%)$ was mixed with $\mathrm{SF}$ solution $(1.5 \mathrm{wt} \%)$ at a volume ratio of 1:4. The solution was then irradiated under ultraviolet light at a wavelength of $365 \mathrm{~nm}$ for six hours. An ultraviolet visible (UV-vis) spectrometer (UV-1800, Shimadz) was used to measure the UV-vis absorption spectrum of the AgNPs. Transmission electron microscopy (TEM, JEM-2100) was used to observe the morphology of the AgNPs, from which the mean diameter of the particles was measured and determined by ImageJ.

\subsection{Preparation of AgNP-loaded SF/CMC sponge}

An equal volume of carboxymethylchitosan (CMC) solution (1.2 wt \%) was supplemented into and mixed with the SF solution containing the AgNPs. After bubbles were removed by centrifugation, an equal volume $(570 \mu \mathrm{L}$ or $3 \mathrm{~mL})$ of the resulting solution was pipetted into each well of 6 -well or 24 - 
well cell culture plates (Costar, Corning Inc.). The solutions were prefrozen at $-20^{\circ} \mathrm{C}$ for 30 minutes, frozen at $-80^{\circ} \mathrm{C}$ overnight, and then lyophilized. The lyophilized sponge was then crosslinked by $1-$ ethyl-3-(3-dimethylaminopropyl) carbodiimide hydrochloride/N-Hydroxysuccinimide, (EDC/NHS, 50 $\mathrm{mM}$ and $12.5 \mathrm{mM}$ ) solution containing $90 \%$ (v/v) ethanol for 12 hours. After crosslinking, the sponge was washed three times with distilled water to remove unreacted substances, and then lyophilized again to obtain the AgNP-loaded SF/CMC sponge. AgNP-null SF and SF/CMC sponges were prepared at the same concentrations of SF and CMC as that of the AgNP-loaded SF/CMC sponge.

\subsection{Scanning electron microscopy (SEM)}

Morphology of the sponges was observed by SEM (S-4800, Hitachi Instruments). The internal structures of the sponges, exposed by cross-sectional dissection, were coated with gold using an ion sputterer (E-1010; Hitachi) at $50 \mathrm{mTorr}$ and $5 \mathrm{~mA}$ for 30 seconds, and viewed by SEM at a voltage of $1.0 \sim 2.0 \mathrm{kV}$.

\subsection{Anti-bacterial activity of the sponges}

The anti-bacterial activity of the sponges against both Gram-positive (S. aureus) and Gram-negative (P. aeruginosa) bacteria was investigated using the Kirby-Bauer disc diffusion method and bacterial suspension assay.

During the Kirby-Bauer disk-diffusion method, $50 \mu \mathrm{L}$ of bacterial suspension at log-phase was uniformly inoculated and cultured on a Mueller-Hinton $(\mathrm{M} \cdot \mathrm{H})$ Agar plate (Oxoid, Thermo Fisher). After a bacterial biofilm formed on the plate, the fully water-absorbed sponge, AQUACEL ${ }^{\circledR} \mathrm{Ag}$ (positive control), and SF/CMC sponge (negative control) were placed on top of the film, and zones of inhibition were observed after 24 hours.

During the bacterial suspension assay, $50 \mu \mathrm{L}$ of bacterial suspension was inoculated overnight in 10 $\mathrm{mL}$ of $\mathrm{M} \cdot \mathrm{H}$ medium under constant shaking at $200 \mathrm{rpm}$ at $37^{\circ} \mathrm{C}$. The bacterial solution was then diluted in $\mathrm{M} \cdot \mathrm{H}$ medium to an O.D. of 0.07 at $600 \mathrm{~nm}$. $400 \mu \mathrm{L}$ of the solution was then added into a test tube filled with $5 \mathrm{~mL}$ of $\mathrm{M} \cdot \mathrm{H}$ medium and containing AgNP-loaded SF/CMC sponge, AQUACEL ${ }^{\circledR} \mathrm{Ag}, \mathrm{SF} / \mathrm{CMC}$ sponge, or no test material (blank control). The test tube was then wrapped in tin foil, and placed at $37^{\circ} \mathrm{C}$ under constant shaking at $50 \mathrm{rpm}$ for 24 hours. Then, $100 \mu \mathrm{L}$ of the bacterial solution was removed from the test tube for O.D. measurement.

\subsection{Water absorption capacity and retention test}

The water absorption capacity (WAC) of the samples was measured using a modified British Pharmacopoeia (BP) method. First, a circular sample, $2 \mathrm{~cm}$ in diameter, was weighed $(W)$ and then immersed in $50 \mathrm{~mL}$ of $\mathrm{BP}$ solution $\mathrm{A}\left(142 \mathrm{mM} \mathrm{NaCl}, 2.5 \mathrm{mM} \mathrm{CaCl}_{2}\right)$ in a $100-\mathrm{mL}$ beaker. After incubation at $37^{\circ} \mathrm{C}$ for one hour, the sample was removed by forceps and weighed again $\left(W^{\prime}\right)$. WAC (\%) was calculated as $\left(W^{\prime}-W\right) / W \times 100 \%$.

The water retention ability (WR) of the samples was tested using a modified method from that described by Liu, et al [10]. Briefly, the fully water-absorbed sample was placed in a desiccator containing silica gel at $37^{\circ} \mathrm{C}$ for 24 hours. The weights of fully water-absorbed samples $\left(W^{\prime}\right)$, samples removed from the desiccator $(W)$, and dry samples $\left(W_{0}\right)$ were recorded. The WR $(\%)$ per hour was calculated as $\left(W^{\prime}-W\right) /\left(W_{0} \times 24\right) \times 100 \%$. 


\subsection{Measurement of water vapor transmission rate}

The water vapor tranmission rate (WVTR) was measured in accordance with the standard ASTM D1653. Briefly, a Perm Cup (Gardner Company, America) was filled to $4 \mathrm{~mm}$ from the top with distilled water and then sealed by the circular freeze-dried sample of $9.5 \mathrm{~cm}^{2}$. The assembly was weighed, kept in an environment of $25 \pm 0.5^{\circ} \mathrm{C}$ and $40 \pm 2 \%$ relative humidity for 24 hours, and then weighed again. WVTR $\left(\mathrm{g} / \mathrm{m}^{2} / \mathrm{h}\right)$ was calculated by the following equation: WVTR $=\frac{\left(W^{\prime}-W\right)}{A \times 24}$, where $W$, and $W$ represent the assembly weight before and after 24 hours of water evaporation, respectively, and $A$ represents the transmission area of the sealing sample.

\section{Results and discussion}

\subsection{Synthesis of AgNPS}

Silk fibroin can reduce $\mathrm{Ag}^{+}$to $\mathrm{Ag}$ by the strong electron donating property of its tyrosine (Tyr) amino acid residue. Meanwhile, UV light irradiation is an effective method to assist the synthesis of AgNPs or $\mathrm{Ag}$ clusters on silk fibers [11]. In this study, $\mathrm{AgNPs}$ were synthesized from $\mathrm{Ag}^{+}$in $\mathrm{SF}$ solution under 6-hours of UV light irradiation. The formation of AgNPs was confirmed by color transition of the solution from colorless to brown, as shown in Figure 1(a). This color transition is correlated with surface plasmon resonance (SPR) of AgNPs. UV-vis absorption spectrum of the solution displays this characteristic SPR band at $\sim 440 \mathrm{~nm}$, as shown in Figure 1(a). As shown in Figure 1(b), the synthesized AgNPs were spherical in shape, and had a mean diameter of $4.9 \pm 1.9 \mathrm{~nm}$, calculated by the average measurement of 100 nanoparticles.

\subsection{Morphology of AgNP-loaded SF/CMC sponge}

Pore structure and porosity are key parameters of a wound dressing which affect the water absorption capacity, water vapor transmission, and modulus of the dressing. Although one of the primary advantages of SF for biomaterial application is its assembly into an insoluble matrix in the
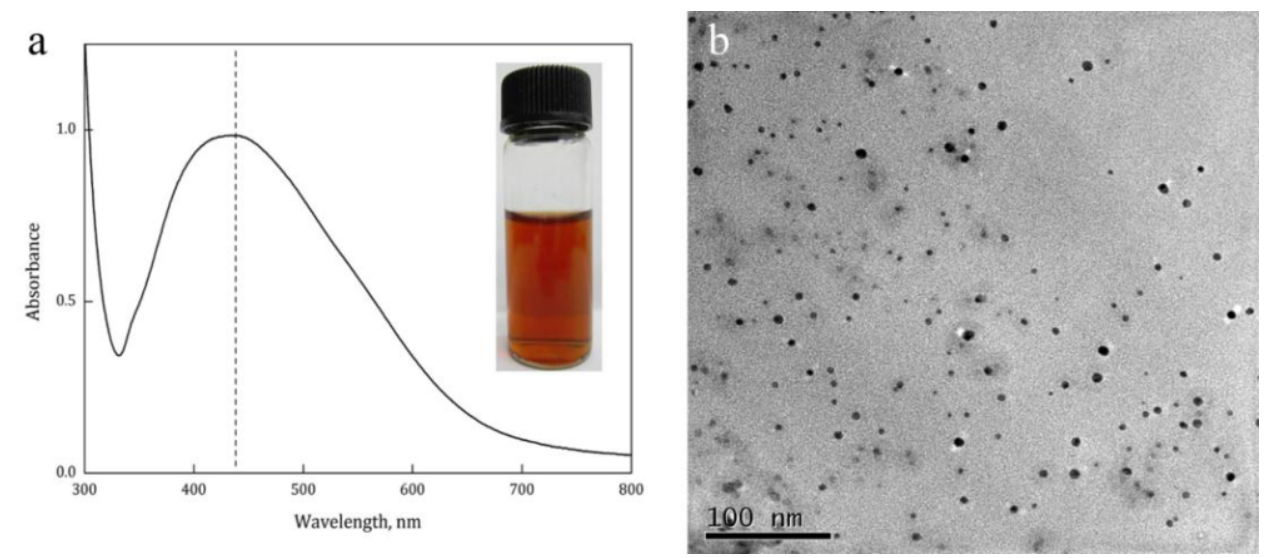

Fig. 1. Synthesis and characterization of AgNPs in SF solution. (a) Image and UV-visible absorption spectrum of silver nanopartcles in SF solution; (b) TEM-observed morphology of AgNPs. 

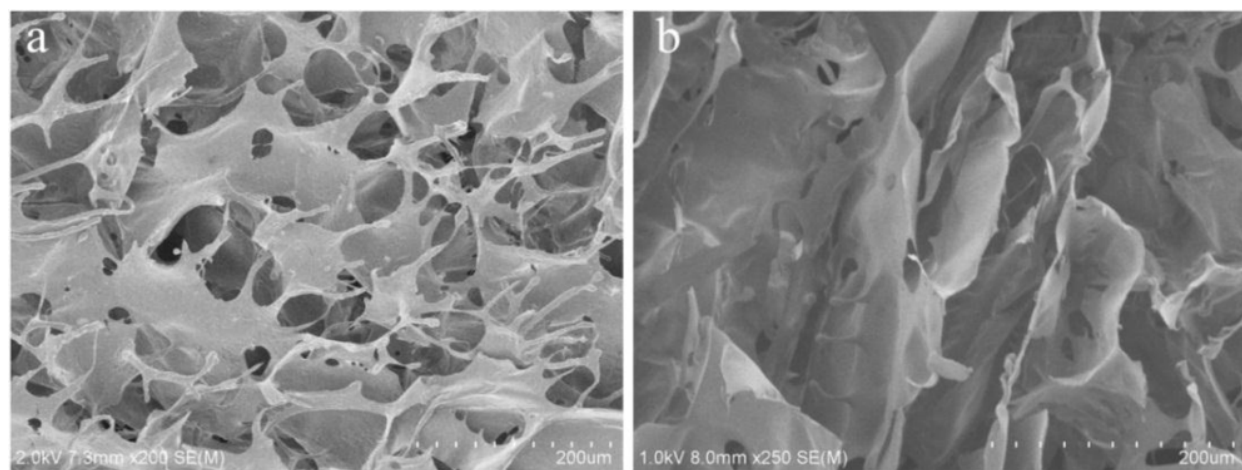

Fig. 2. SEM micrographs of (a) AgNP-loaded SF/CMC sponge; and (b) pure SF sponge.
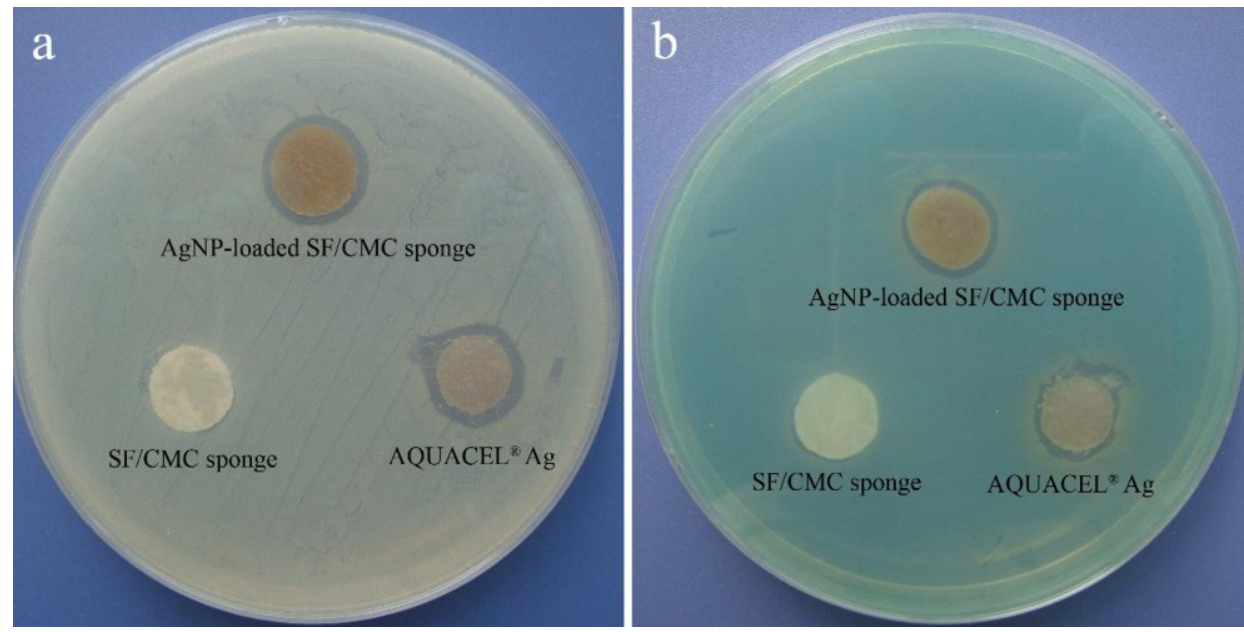

Fig. 3. Anti-bacterial properties against (a) S. aureus and (b) P. aeruginosa using the Kirby-Bauer disk diffusion method (24 hours after treatment).

absence of chemical crosslinkers, this structural transformation is intense, and thus difficult to modulate in order to fabricate SF-based scaffolds with controlled silk II ( $\beta$-sheet) formation [12]. In this study, SF was combined with CMC to reduce the silk II content of SF. The AgNP-loaded $\mathrm{SF} / \mathrm{CMC}$ sponge had a porous structure, as shown in Figure 2(a), while the homogeneous SF sponge had an irregular sheet-like structure which lacked porosity, as shown in Figure 2(b). Moreover, no visible phase separation was observed in AgNP-loaded SF/CMC sponges, as shown in Figure 2(a). SF was combined with other polymers, such as collagen, gelatin, and chitosan, in order to fabricate versatile biomaterials with regard to biocompatibility and tunable physical properties [13-15]. The incorporation of another polymer can improve the porosity of the integral materials, partially accomplished by interrupting the secondary structural transformation of SF.

\subsection{Anti-bacterial activity of AgNP-loaded SF/CMC sponge}

The anti-bacterial activity of the composite material was tested by disk diffusion and suspension. As shown in Figures 3(a) and 3(b), results of the disk diffusion analysis demonstrated the formation of clear rings around AgNP-loaded SF/CMC sponges and AQUACEL ${ }^{\circledR}$ Ag after 24 hours, whereas no 
zone of inhibition was observed around AgNP-null SF/CMC sponges. To further measure the antibacterial activity of the AgNP-loaded SF/CMC sponge, suspension assays were conducted by determining the O.D. of the bacterial solution in the presence and absence of the sponge. For the two bacterial strains, solutions of the sample treated with the AgNP-loaded SF/CMC sponge was more transparent than that treated with an SF/CMC sponge and the blank control, as shown in Figures 4(a) and 4(b). The O.D. of the sample treated by the AgNP-loaded SF/CMC sponge was significantly lower than that of the samples treated by the AgNP-null SF/CMC sponge and the blank control, as shown in Figures 4(c) and 4(d). The O.D. of the sample treated by the AgNP-loaded SF/CMC sponge was lower than that treated by AQUACEL ${ }^{\circledR}$ Ag, as shown in Figures 4(c) and 4(d). Both the disk diffusion method and bacterial suspension assay results showed a similar trend regarding the anti-bacterial activity of the AgNP-loaded SF/CMC sponge. It is worth noting that the AgNP-loaded SF/CMC sponge was more effective against $P$. aeruginosa, a bacterial strain particularly known for its drug resistance.
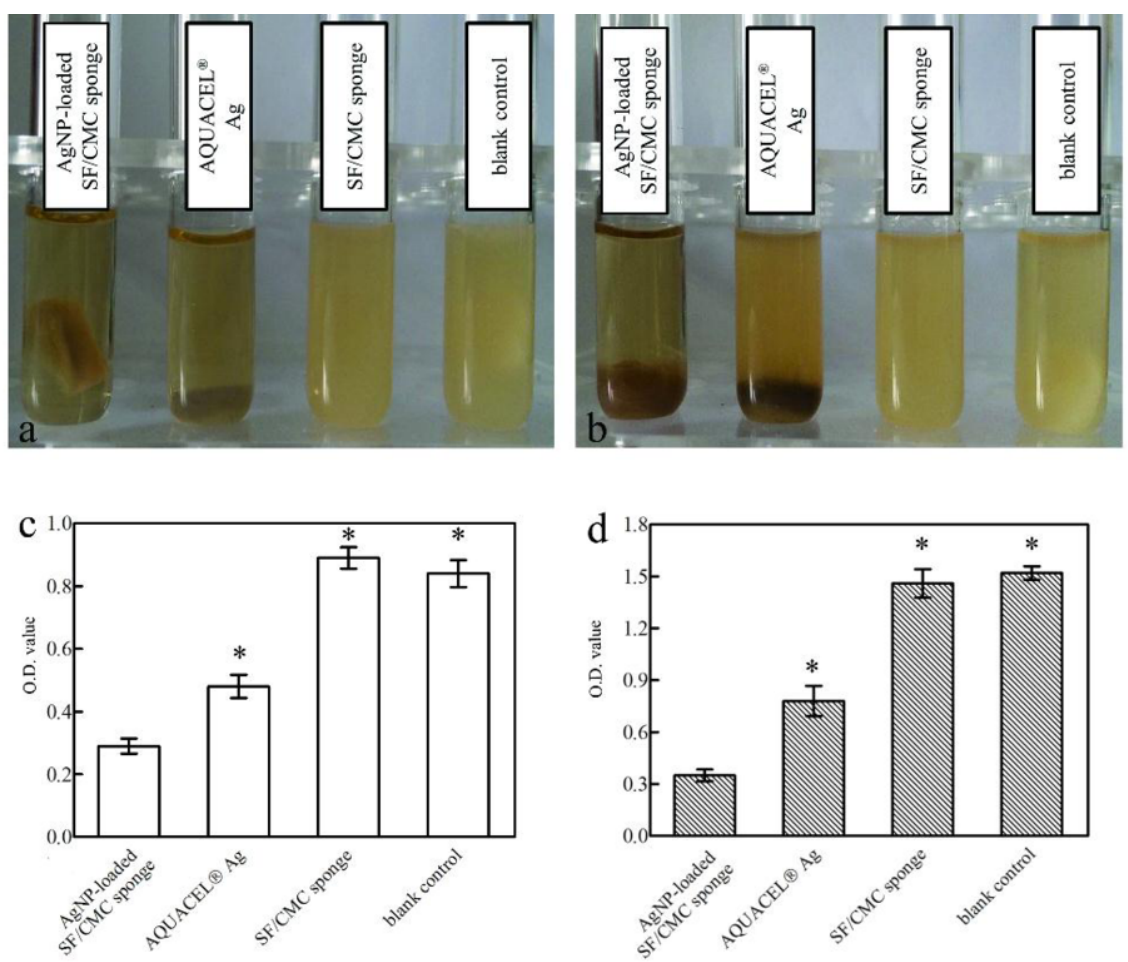

Fig. 4. Anti-bacterial activity against $S$. aureus and P. aeruginosa determined by bacterial suspension assay. (a) Sample images of $S$. aureus solutions; (b) sample images of P. aeruginosa solutions; (c) O.D. values of S. aureus solutions; (d) O.D. values of $P$. aeruginosa solutions (24 hours after treatment). * represents statistical significance, in comparison to AgNPloaded SF/CMC sponge.

Table 1

WAC, WR, and WVTR of sponges

\begin{tabular}{llll}
\hline & WAC $(\%)$ & WR $(\%)$ & WVTR $\left(\mathrm{g} / \mathrm{m}^{2} / \mathrm{h}\right)$ \\
\hline SF sponge & $623 \pm 36$ & $43.3 \pm 3.8$ & $163.64 \pm 22.98$ \\
SF/CMC sponge & $1487 \pm 157$ & $83.8 \pm 5.2$ & $109.23 \pm 19.36$ \\
AgNP-loaded SF/CMC sponge & $1496 \pm 149$ & $82.6 \pm 5.5$ & $115.43 \pm 18.53$ \\
\hline
\end{tabular}




\subsection{WAC and WR}

WAC and WR are key material properties that determine the performance of biologic dressings in maintaining a moist environment for wound healing. The WACs of the SF/CMC sponge and the AgNP-loaded SF/CMC sponge were comparable and more than twice that of the SF sponge (see Table 1). Similarly, water retention ratios of the SF/CMC sponge and the AgNP-loaded SF/CMC sponge were also comparable and approximately twice that of the SF sponge (see Table 1). These results indicate that the presence of CMC improves the water absorption and retention ability of SF/CMC composite sponges, and is not weakened by AgNP synthesis and loading.

\subsection{WVTR}

An appropriate moist environment is important for the facilitation of wound healing. Insufficient removal of wound exudate leads to wound edema and increased risk of infection, and excessive water loss leads to wound hydration. In this study, the average WVTRs for AgNP-loaded SF/CMC and $\mathrm{SF} / \mathrm{CMC}$ sponges were 115.43 and $109.23 \mathrm{~g} / \mathrm{m}^{2} / \mathrm{h}$, respectively, both lower than that of the SF sponge (see Table 1). The AgNP-loaded SF/CMC sponges in this study had a WVTR of $111.92 \mathrm{~g} / \mathrm{m}^{2} / \mathrm{h}$, which is similar to that of gelatin, alginate, and borax-based wound dressings, and is appropriate for maintaining a fluid balance on the wound bed [16].

\section{Conclusion}

An AgNP-loaded SF/CMC sponge was developed for use as an anti-bacterial wound dressing. The sponge demonstrated anti-bacterial activity against $S$. aureus and $P$. aeruginosa, with anti- $P$. aeruginosa activity higher than that of AQUACEL $\mathbb{A} \mathrm{Ag}$, a commercial $\mathrm{Ag}^{+}$dressing. In addition, the sponge displayed improved water absorption capacity and retention abilities, and an optimal water vapor transmission rate. This AgNP-loaded SF/CMC sponge is a promising wound dressing candidate for the prevention of wound infection and the simultaneous promotion of wound healing.

\section{References}

[1] J.S. Boateng, K.H. Matthews, H.N. Stevens and G.M. Eccleston, Wound healing dressings and drug delivery systems: A review, Journal of Pharmaceutical Sciences 97 (2008), 2892-2923.

[2] L.A. Barajas-Nava, J. López-Alcalde, M. Roqué i Figuls, I. Sola and X. Bonfill Cosp, Antibiotic prophylaxis for preventing burn wound infection, the Cochrane Database of Systematic Reviews 6 (2013), CD008738.

[3] F. De Cicco, A. Porta, F. Sansone, R.P. Aquino and P. Del Gaudio, Nanospray technology for an in situ gelling nanoparticulate powder as a wound dressing, International Journal of Pharmaceutics 473 (2014), 30-37.

[4] B.D. Kevadiya, S. Rajkumar, H.C. Bajaj, S.S. Chettiar, K. Gosai, H. Brahmbhatt, A.S. Bhatt, Y.K. Barvaliya, G.S. Dave and R.K. Kothari, Biodegradable gelatin-ciprofloxacin-montmorillonite composite hydrogels for controlled drug release and wound dressing application, Colloids and Surfaces B: Biointerfaces 122 (2014), 175-183.

[5] L.G. Ovington, The truth about silver, Ostomy Wound Management 9A (2004), 1S-10S.

[6] J.J. Castellano, S.M. Shafii, F. Ko, G. Donate, T.E. Wright, R.J. Mannari, W.G. Payne, D.J. Smith and M.C. Robson, Comparative evaluation of silver-containing antimicrobial dressings and drugs, International Wound Journal 4 (2007), $114-122$.

[7] M. Rai, A. Yadav and A. Gade, Silver nanoparticles as a new generation of antimicrobials, Biotechnology Advances 27 (2009), 76-83.

[8] J. Verbelen, H. Hoeksema, A. Heyneman, A. Pirayesh and S. Monstrey, Aquacel ${ }^{\circledR}$ Ag dressing versus Acticoat ${ }^{\mathrm{TM}}$ 
dressing in partial thickness burns: a prospective, randomized, controlled study in 100 patients. Part 1: burn wound healing, Burns 40 (2014), 416-427.

[9] M.R. Reithofer, A. Lakshmanan, A.T. Ping, J.M. Chin and C.A. Hauser, In situ synthesis of size-controlled, stable silver nanoparticles within ultrashort peptide hydrogels and their anti-bacterial properties, Biomaterials 35 (2014), $7535-7542$.

[10] T.Y. Liu, S.Y. Chen, Y.L. Lin and D.M. Liu, Synthesis and characterization of amphiphatic carboxymethyl-hexanoyl chitosan hydrogel: water-retention ability and drug encapsulation, Langmuir 22 (2006), 9740-9745.

[11] Z. Lu, M. Meng, Y. Jiang and J. Xie, UV-assisted in situ synthesis of silver nanoparticles on silk fibers for antibacterial applications, Colloids and Surfaces A: Physicochemical and Engineering Aspects 447 (2014), 1-7.

[12] O. Rathore and D.Y. Sogah, Nanostructure formation through $\beta$-sheet self-assembly in silk-based materials, Macromolecules 34 (2001), 1477-1486.

[13] L. Chen, J. Hu, J. Ran, X. Shen and H. Tong, Preparation and evaluation of collagen-silk fibroin/hydroxyapatite nanocomposites for bone tissue engineering, International Journal of Biological Macromolecules 65 (2014), 1-7.

[14] W. Xiao, J. He, J.W. Nichol, L. Wang, C.B. Hutson, B. Wang, Y. Du, H. Fan and A. Khademhosseini, Synthesis and characterization of photocrosslinkable gelatin and silk fibroin interpenetrating polymer network hydrogels, Acta Biomaterialia 7 (2011), 2384-2393.

[15] G.J. Lai, K.T. Shalumon, S.H. Chen and J.P. Chen, Composite chitosan/silk fibroin nanofibers for modulation of osteogenic differentiation and proliferation of human mesenchymal stem cells, Carbohydrate Polymers 111 (2014), 288-297.

[16] B. Balakrishnan, M. Mohanty, P.R. Umashankar and A. Jayakrishnan, Evaluation of an in situ forming hydrogel wound dressing based on oxidized alginate and gelatin, Biomaterials 26 (2005), 6335-6342. 